\title{
Some psycholinguistic aspects of person perception*
}

\author{
NORMAN H. ANDERSON and LOLA L. LOPES \\ University of California, San Diego, La Jolla, California 92037
}

\begin{abstract}
Adjective-noun combinations in person perception are analyzed from an integration-theoretical view, with special reference to judgments of likableness, occupational proficiency, and social value. Different theoretical considerations are shown to apply to these three judgment dimensions, but all can be conceptualized in terms of the valuation and integration operations of integration theory. Experimental support for this conceptual analysis is given for likableness and occupational proficiency. Advantages of the integration-theoretical analysis over congruity formulations are pointed out.
\end{abstract}

Many studies of person perception stand in the border region where judgment theory merges into psycholinguistics. Much of our information about other people is conveyed by words, and these words affect our perceptions and opinions about them. The study of word combinations is, therefore, of direct relevance to person perception.

This relevance is reciprocal. A moment's reflection will show how broadly the vocabulary of person perception pervades the description of the animate and inanimate world. Personification and animism illustrate how our psychological mechanisms of person perception are transposed directly to object perception in general. We see both the human and the nonhuman worlds in similar terms.

Previous experimental work in person perception has been largely limited to one specific type of word combination: how two adjectives combine to determine a total impression. This article considers a different question: how an adjective and a noun combine. Very little is known about this. Two pioneering papers on this problem, by Osgood, Suci, and Tannenbaum (1957, Chapter 7) and by Rokeach and Rothman (1965), used one or another form of congruity-type principle. The present approach is based on a principle of information integration that has several advantages over the congruity approach.

\section{INFORMATION INTEGRATION THEORY}

Information integration theory has worked well on the adjective-adjective problem, and a good deal of that knowledge can be transferred directly to the adjective-noun problem. However, the adjective-noun problem has added complexities that require more detailed conceptual analysis. This conceptual analysis will be given following a few summary comments on integration theory.

*This work was supported by NSF: Grants GB-21028 and GS-36918 and facilitated by grants from the National Institute of Mental Health to the Center for Human Information Processing, University of California, San Diego. We wish to thank Gregg Oden and Raymond Smith for their helpful comments.
Integration theory considers the person to be an integrator of stimulus information. When judging a target object, the person first evaluates each piece of stimulus information in the context of the dimension of judgment. He then integrates this evaluated information to obtain a single judgment.

\section{Valuation}

Each stimulus has a theoretical representation in terms of two parameters, a scale value (s) and a weight (w). The scale value represents the position of the stimulus along the judgment dimension. The weight represents the relevance and importance of the stimulus to the dimension of judgment.

As illustrated below, both the value and the weight parameters depend on the dimension of judgment. Thus, in any judgment situation, the person must begin by evaluating the stimuli to get the stimulus parameters that apply to the particular judgment he is to make.

\section{Integration}

The integration operation governs the combination of the separate pieces of stimulus information. The stimuli might be averaged together, for instance, or they might be multiplied. Integration theory relies heavily on such simple algebraic models of the integration operation. These models, combined with factorial stimulus designs, lead to very simple analyses. In particular, they allow functional measurement of scale values and, under certain circumstances, of weights. More detailed treatments of integration theory are given in Anderson (1971a, 1973, 1974).

\section{CONCEPTUAL ANALYSIS}

The adjective-noun problem has a number of interesting complexities. Especially important is the dimension of judgment. The same target person may be judged on various dimensions: how likable he is, how sociable, how good at his job, how likely to enjoy baseball. And the answers will differ not only in their 
final value but also in the processes by which the judgment is obtained.

Three judgment dimensions have special importance in person perception: likableness, proficiency, and social value. They will be considered in detail for two reasons. First, they are easily confounded in judgment situations that ask for responses on a simple good-bad dimension. Second, they are good examples of how various the subprocesses underlying overt judgment can be.

The discussion will focus on the case of one or more adjectives linked with an occupation noun like lawyer or plumber. However, the same points apply to adjective-noun combinations where the noun defines some social role, like mother or friend.

\section{Likableness}

Judgments of likableness have an unusual property. The occupation noun carries information of the same quality as the adjective. A teacher, for example, is expected to have certain characteristics that bear as directly on likableness as does the stimulus adjective. According to integration theory, therefore, the occupation and the adjective should be averaged to produce the judgment.

\section{Occupational Proficiency}

Judgments of occupational proficiency are quite different from judgments of likableness. In this case, the occupation noun itself defines the dimension of judgment. The value and weight of the adjective are defined along that dimension, and an inference is required to determine them. Clearly, the adjective and the noun cannot be integrated as equivalent quantities.

Two main classes of adjectives may be distinguished. The first class consists of adjectives that lie directly on the proficiency dimension itself. Capable and incompetent, for example, should act directly as proficiency quantifiers. For this class, the valuation operation yields the judgment and no integration is required.

The second class consists of words that are more or less occupation specific. Examples are gracefulness in a dancer and persuasiveness in a lawyer. The weight parameter of such adjectives depends strongly on the occupation; gracefulness would have less weight for a statistical clerk than for a dancer.

Some words fall in both classes. Words such as intelligent and resourceful might be thought to act directly as quantifiers on the proficiency dimension. An intelligent or resourceful person would be expected to do better at his own occupation regardless of what it is. Nevertheless, neither trait would be weighted as strongly in a garbage collector as in a lawyer. It is possible, therefore, that some words have a dual function.

Strictly speaking, proficiency judgments involve a hidden integration operation, particularly for adjectives of the second class. Once the adjective has been evaluated, it must be integrated with the initial impression, an internal stimulus that corresponds to a generalized proficiency expectation in the absence of specific given information. This point will be ignored here, but it has theoretical importance in certain applications.

\section{Social Value}

Judgments of social value demonstrate some potential complexities of adjective-noun integration. They also demonstrate the multidimensional structure of judgment. A person's value to society depends both on the social value of his occupation, which is an evaluative judgment like likableness, and on his proficiency at that occupation. Thus, two preliminary judgments must precede the final judgment of social value.

The operation integrating these two preliminary judgments may also be of interest. It is not unreasonable to suppose that a multiplying model holds, with the proficiency value of the adjective multiplying the social value of the noun. That would produce judgments of low social value when either the proficiency or the social value of the occupation is low.

\section{EXPERIMENTAL DESIGN CONSIDERATIONS}

Study of the adjective-noun problem depends critically on certain problems of experimental procedure. These are not merely technical but relate closely to the conceptual analysis.

\section{Response Measure}

It is of first importance to have a clean response measure. To ask for judgments of happy nurse on a generalized good-bad dimension or on social desirability is to ask for trouble. Such responses will be hodgepodge composites of all three of the judgment dimensions that have been considered just above. A hodgepodge measure may have uses, but an adequate theoretical structure needs a clean delineation of the various dimensions of judgment.

This is a basic criticism of much of the work that has used the semantic differential. Osgood et al (1957), for example, used responses averaged over three rating scales: valuable-worthless, admirable-deplorable, and good-bad. Rokeach and Rothman (1965) used the same scales in the same way.

The semantic differential obscures the dimension of judgment almost as a matter of principle. It factors out the denotative components, and it appears to agglomerate various evaluative components that should be kept separate. The semantic differential may have useful applications, but its role in judgment theory seems quite limited.

Take clever thief as an example. Is a clever thief better 
or worse than a dull thief? The clever thief is probably more likable personally and no doubt better at his job. But, being better at his job, he would have less social value. Evaluation of clever thief on a generalized good-bad scale confounds all three judgment dimensions.

These questions can be asked separately with simple rating scales that explicitly define each particular dimension of judgment. Although there may be judgment dimensions that are intrinsically intertwined, as perhaps with social value, such confounding should ordinarily be avoided when possible.

\section{Parallelism Predictions}

The experiment reported below uses an adjective by adjective by noun factorial design. Each stimulus person is described by his occupation role and by two personality traits.

This design has an important function. Existing evidence for judgments like likableness and job proficiency indicates that the two adjectives will be averaged within the context of a given noun. Under certain conditions, therefore, the data for any particular noun should plot as a set of parallel curves. This graphical prediction of parallelism has a simple test: The Adjective by Adjective interaction should be nonsignificant.

Furthermore, if the noun is averaged with the adjectives, as is expected for likableness judgments, the parallelism should extend across noun cells. In the complete adjective by adjective by noun design, there should then be no significant interactions. Of course, if the adjective valuation depends on the noun, as in judgments of job proficiency, interaction between adjectives and noun would be expected.

\section{Differential Weighting}

The simple parallelism prediction requires that the stimuli within each factor of the stimulus design have equal weight. It is a fortunate fact that this equal-weighting condition is approximately correct for adjective stimuli with the likableness judgment. The simplicity and directness of the parallelism property have made it a key tool in the development of integration theory.

Perhaps no other judgment dimension in person perception meets this requirement in a general way. Graceful and mathematical have quite different weights for the occupational proficiency of a dancer. The same applies more generally to judgments of personality characteristics other than likableness. In all these judgments, differential weighting must be expected (e.g.. Lampel \& Anderson, 1968; Oden \& Anderson, 1971; Warr \& Smith, 1970).

Unfortunately, differential weighting destroys the simple parallelism property. This complicates the analysis, but two approaches are feasible. One is to consider the qualitative features of the data: rough estimates of the weight parameters can provide directional predictions of deviations from parallelism (Anderson, 1971a). Another is to use Chandler's (1969) STEPIT program, which can estimate both weights and scale values (e.g., Anderson, 1972; Leon, Oden \& Anderson, 1973). However, the latter requires a fairly large design and may not be well suited for testing goodness of fit.

A different method was used here in hope of retaining equal weighting. Each adjective factor in the design was defined by a single adjective; the levels of the factors were obtained by attaching an adverbial quantifier to the adjective. (This is illustrated in Fig. 1.) Thus, the adjective for a given factor defined a single information dimension; hopefully, it also set the weight of that dimension in the judgment. The adverbial levels of the adjective served to specify location on the adjective dimension, thus defining the scale value.

\section{EXPERIMENTAL METHOD}

This experiment was designed as an initial test of the integration-theoretical analysis given above. The main goal was to assess the potential usefulness of the model for the study of the valuation process. Two of the three dimensions discussed above, likableness and job proficiency, were studied.

Stimulus persons were described by two personality traits and an occupation role in an adjective by adjective by noun design. Ss judged these person descriptions on two rating scales: likableness and occupational proficiency.

\section{Stimuli for Judgments of Likableness}

Each person description for this part of the experiment consisted of an occupation noun modified by two adverb-adjective phrases. The overall design was a 4 by 2 by 3 factorial that can be understood most simply by referring forward to Fig. 1. The four occupation nouns were lawyer and plumber, both specified as male. and dancer and statistical clerk. both specified as female. The modifying phrases formed a 2 by 3 design, in which the row stimuli were two levels of kind (extremely and averagely) and the column stimuli were three levels of humorous (very, moderately, and not very). In addition, a second stimulus replication was used with kind and humorous replaced by polite and cheerful, respectively. Altogether. therefore. there were 48 person descriptions in this part of the experiment.

\section{Stimuli for Judgments of Occupational Proficiency}

This part of the experiment used the same kind of person descriptions as just described. namely, an occupation noun modified by two adverb-adjective phrases. However, different modifying phrases were used for lawyer and plumber, on the one hand, and for dancer and statistical clerk, on the other hand. The overall design can be understood most simply by referring forward to Fig. 2. For lawyer and plumber, the modifying phrases made up three 2 by 3 designs. In all cases, the row stimulus was two levels of reliahle (extremely and averagely). Lach 2 by 3 design was based on a different column adjective. persuasive, mechanical, and musical, as listed in lig. 2. The levels of each column adjective were again given by the three adicrbial yuantifiers, leri, moderately, and not ver

Lxactly the same procedure was used for the other pair of 


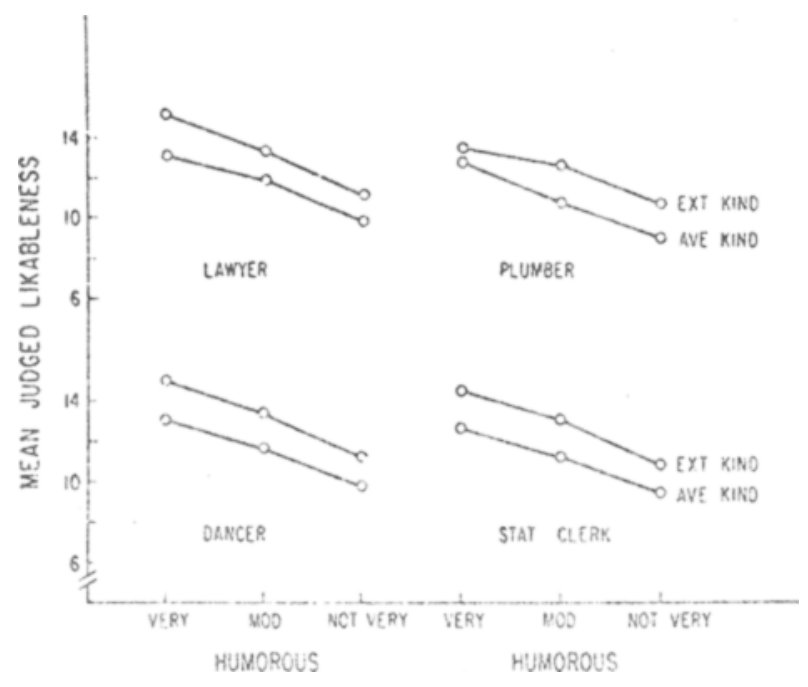

Fig. 1. Mean judgments of likableness of persons described by their occupation and two personality traits. Traits formed a 2 by 3 design with two row levels, extremely and averagely kind. and three column levels, very, moderately, and not very humorous. Data averaged over a second stimulus replication in which kind and humorous were replaced by cheerful and polite, respectively.

occupations, dancer and statistical clerk. The row adjective was changed to dependable, and the three column adjectives were changed to graceful, mathematical, and religious. Altogether, therefore, there were 72 person descriptions in this part of the experiment.

\section{General Procedure}

Each person description was typed on an index card, and these were shown one at a time. Ss responded by placing a pin on a $20 \mathrm{~cm}$ graphic rating scale that was read to the nearest millimeter.

Judgments of likableness were made first. In this case, S was instructed to suppose that he would be meeting the person at some social gathering and to estimate how much he would like to meet and talk with such a person. For these judgments, the deck of 48 descriptions was shuffled separately for each replication for each $\mathrm{S}$.

Judgments of occupational proficiency were made in the second part of the experiment. In this case, $S$ was instructed that likableness was irrelevant and that the judgment should be made on how well the person would do his job. Since job proficiency is specific, the 18 descriptions for each occupation were presented as a group, in separate shuffled order for each replication for each $\mathrm{S}$.

Preliminary practice was given for each type of judgment. This practice included the usual end-anchor stimuli, in which the modifying phrases were more extreme, either positively or negatively, than those used in the main designs. These anchor stimuli help set out the total range of stimuli. They also hold down the ends of the rating scale, so that the data of interest come from the interior of the scale (Anderson, 1974). Following practice, the deck of descriptions was presented twice in succession, so that each $S$ made two responses to each description. The means below are averages over these two replications.

Ss were 40 students from introductory psychology who served as part of a course requirement. They were tested individually in sessions that lasted about $1 \mathrm{~h}$.

\section{RESULTS}

\section{Likableness}

The likableness data are plotted in Fig. 1, one panel for each occupation noun as listed. Also listed are the adverb-adjective phrases that modified each occupation noun.

Two tests of information integration are at issue in Fig. 1, both of which rest on a prediction of parallelism. The first test concerns the adjective-adjective integration. As can be seen, the two curves for each occupation are approximately parallel, as predicted by the integration model. The statistical test of parallelism is the Row by Column interaction term in the analysis of variance and that was nonsignificant $[F(2,78)=1.40]$, in support of the model. More detailed analyses showed significant interactions for three occupations in the cheerful-polite replication. These are ieflected in the mild nonparallelism visible in Fig. 1 for lawyer, plumber, and statistical clerk. These interactions were small and nonsystematic and do not seem to require much qualification of the conclusion that the two adverb-adjective phrases are integrated by a simple averaging rule.

The second test concerns the adjective-noun integration. The main effect of occupation was significant $[\mathrm{F}(3,117)=6.32]$, but it did not interact with either the row or the column adjective. Each of these interactions also corresponds to a parallelism prediction. For example, if the two curves for each occupation in Fig. 1 were averaged to yield a single curve, these four occupation curves would be essentially parallel. These results, therefore, imply that adjective and noun are averaged as equivalent quantities, as originally hypothesized.

This result on adjective-noun integration is quite interesting. It should be emphasized, however, that it does not hold for all judgment dimensions, as will be seen in the next subsection, and its generality is unknown even for the likableness dimension. To the extent that this result generalizes, however, the simpler adjective by noun design will be adequate. Such two-way designs still provide a test of the parallelism prediction. Also, the marginal means would yield functional scales of the noun and adjective stimuli.

\section{Occupational Proficiency}

Figure 2 plots the data on occupational proficiency. The two curves in each panel represent a 2 by 3 design for the two adverb-adjective phrases that modified each occupation. For simplicity, only the data for lawyer and plumber will be discussed in detail; essentially, the same picture is shown by the data for dancer and statistical clerk in the lower half of Fig. 2.

For lawyer and plumber, the same descriptive traits 
were used. The three column adjectives were chosen so that persuasive would be relevant for lawyer but not for plumber, mechanical would be relevant for plumber but not for lawyer, and musical would be relevant for neither. Since relevance affects the weight parameter of the averaging model, this choice of stimuli should control the shape of the curves in Fig. 2.

Three features of Fig. 2 require comment. First, the data for each occupation clearly exhibit the differential weighting of the adjectives. The slope of each curve is a measure of the relevance or weight of the corresponding column adjective. For lawyer, at the top of the figure, the curves for mechanical and musical are flat. That reflects the irrelevance of these two traits to judgments of occupational proficiency for lawyer. On the other hand, persuasive has a substantial slope since it is relevant. The same picture can be seen for each of the other three occupations.

Second, the data support an averaging model as opposed to an adding model. The two row curves are closer together when the column adjective is relevant, farther apart when the column adjective is irrelevant, and this effect is significant $[F(2,78)=6.04]$. This effect is predicted by the averaging model, but it is contrary to an adding model. The averaging model implies that the distance between the two row curves in any panel varies inversely with the weight of the column adjective. Because the stimulus weights must sum to unity in each person, greater relevance of the column adjective necessarily reduces the relative importance of the row adjective. The adding hypothesis lacks this restriction on the sum of the weights and does not account for this aspect of the data.

The third feature of Fig. 2 relates to the parallelism prediction. Under the equal-weighting assumption, the averaging model predicts that each of the 12 pairs of curves should be parallel. This is true for the irrelevant adjectives, of course, but there is a clear divergence for each of the four relevant adjectives. Although this divergence is not large, it was reliable as shown by the overall Row by Column interaction for these four cases $[\mathrm{F}(2,78)=21.98]$.

The simplest explanation of this divergence is that unequal weighting remained in spite of use of quantified adjectives. The direction of the nonparallelism would indicate greater weighting for the lower levels of each column adjective, in accord with previous work (e.g., Lampel \& Anderson, 1968; Oden \& Anderson, 1971). Such differential weighting makes sense for judgments of occupational proficiency. If the dancer is not very graceful, for example, it may not make much difference how dependable she is. But as her gracefulness begins to exceed a minimum level, the trait of dependability takes on increasing importance. This integration rule is usually considered as a conjunctive strategy, and such a pattern of judgment can be captured by the averaging model with differential weighting (Anderson, 1971a, 1974).

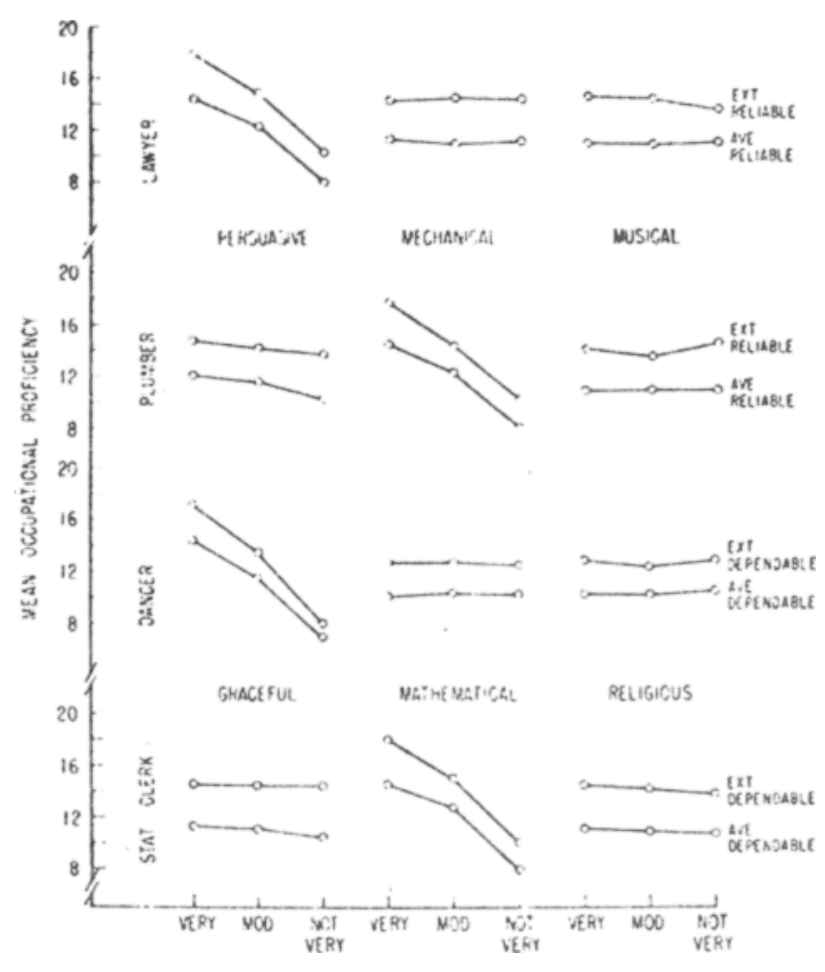

Fig. 2. Mean judgments of occupational proficiency of persons described by their occupation and two personality traits. Format as in Fig. 1.

\section{RELATED WORK}

\section{Congruity Formulations}

The problem of adjective-noun integration has been considered most directly by Osgood et al (1957) and by Rokeach and Rothman (1965). Both papers use an averaging-type model and, in that respect, are similar to the present formulation. However, their basic theoretical postulate is quite different, as both are based on a congruity principle. In contrast, the present formulation is based on a principle of information integration.

The difference between these two principles can be illustrated in the example of punctual nurse judged on likableness. In the present formulation, this phrase contains two pieces of information about the person. Both pieces of information bear on likableness, and they are integrated by averaging to produce the judgment. Thus, the operative principle is simply one of combining information.

The congruity approach is quite different conceptually. Osgood et al assume that the adjective and noun, like all words of unequal scale value, are incongruous with each other. Pressure to reduce this incongruity results in a compromise between the values of the adjective and the noun. Rokeach and Rothman use a variant in which the incongruity is between the adjective-noun phrase, on the one hand, and the adjective, the noun, or both, on the other hand. 
An incongruity interpretation does not seem very realistic for punctual nurse, or even for unpunctual nurse which contains affective inconsistency. In neither case is there any obvious incongruity that needs to be resolved.

The integration-theoretical interpretation is simpler and more plausible. Also, it has a considerable body of experimental support. Informational inconsistencies can arise that do require active resolution, as will be discussed below. However, most information seems to be integrated by simpler processes. This problem is discussed further in Anderson (1971a, pp. 188-189, 1974).

The specific averaging model used by Osgood et al places certain a priori restrictions on the weight parameters (see Anderson, 1971a, pp. 188-189; Rosenberg, 1968). These have proven to be so restrictive that, even with various corrections, the model has done quite poorly in quantitative testing.

The averaging model used by Rokeach and Rothman is more similar to that developed in integration theory because it does not place a priori restrictions on the weight parameters. Indeed, Rokeach and Rothman claim that the weight parameters (but not the scale values) are uniquely and configurally determined in each combination. They conclude, therefore, that the response to the combination cannot be predicted from knowledge of the separate components alone because the weights are unique to each combination. This conclusion forms the basis of their main objection to Osgood et al (Rokeach \& Rothman, 1965, pp. 141-142). If Rokeach and Rothman were correct, then the present integration model would not hold.

Another objection to Rokeach and Rothman's formulation is that their averaging model does not apply io adjective-noun combinations such as irresponsible father which are judged worse than irresponsible. In such cases, Rokeach and Rothman abandon the averaging model and invoke a process of "over-assimilation." In contrast, integration theory uses the same basic integration rule in all cases, since either differential weighting or valuational interaction could account for "over-assimilation."

At best, the congruity formulations have limited scope. They deal with evaluative judgments on a generalized good-bad scale and are ill-equipped to handle judgments on specific dimensions, such as occupational proficiency. Even on the good-bad evaluative scale, the judgmental structure of the semantic differential would confound likableness and occupational proficiency, not to mention social value. These different judgment dimensions should ordinarily be kept distinct, as noted above.

\section{Other Related Work}

A few recent experiments also deserve brief mention. Lampel and Anderson (1968) showed that adjective information and photograph information were integrated by an averaging rule. This result agrees with the present data on adjective-noun integration for the likableness dimension. This paper also gave a critical test between the adding and averaging rules. At the same time, it showed that the weight parameter of the photograph varied inversely with its scale value.

An interesting paper by Gollob (1968) studied evaluative judgments of men described by sentences such as "The considerate man helps criminals." This sentence contains two pieces of information about the man, the adjective "considerate" and the predicate "helps criminals." The simplest integration model would assume that these two pieces of information are averaged or added. Gollob assumed an adding model and adopted analysis of variance procedure to test it. The model did fairly well, but the discrepancies were significant and it is not clear that they were not serious.

One interpretation of the discrepancies from Gollob's adding model is that an averaging rule actually applies (see also Gollob \& Lugg, 1973). Inspection of Gollob's Table 1 suggests that the various predicates had different weights, and that would produce statistical interaction if adjective and predicate are averaged rather than added. It is also possible that configural evaluation is involved, as in the analogous impression dyads (Anderson, 1973, Section III.G.1). Helping criminals may be meritorious in a considerate man, but it is certainly evil in an evil man.

Although Gollob did not study adjective-noun integration, his work was extended by Heise (1969), who varied the subject of the sentence, as well as the verb and object, and obtained judgments of all three components in each sentence. Heise claimed support for an additive integration of subject and predicate, which would be consistent with the present data on likableness. Unfortunately, Heise reverted to multiple regression analysis without allowing for fallible predictors and without providing a test of goodness of fit, which is usually mandatory in this kind of work (Anderson, 1972; Birnbaum, 1973; see also Shanteau \& Anderson, 1969 , p. 321). Indeed, Heise's claim that verb-object integration followed a simple multiplying rule is not consistent with Gollob's more careful analysis that found two factors in this interaction.

Despite these reservations and uncertainties, the sentence task studied by Gollob and Heise has considerable interest. Application of functional measurement methodology may help clear up some of the uncertainties.

Jaspars, Rommetveit, Cook, Havelka, Henry; Herkner, Pecheux, and Peeters (1971) modified the usual order effect task in person perception by including an occupation noun either at the beginning or at the end of the list of trait adjectives. The impression response showed recency. Noun location was not significant, though the impression of the person was somewhat more favorable when the defining noun followed the 
adjectives. Unfortunately, this work used the semantic differential to measure the impression, so the results are not easy to interpret. However, the simplest hypothesis would be that the noun is averaged in with the adjectives, as in the likableness data of Fig. 1. Since the nouns were apparently more favorable than the average adjectives, and since recency was found, a more favorable impression would be expected when the noun came at the end. The observed result was in that direction, though not significant. Recall data were also obtained which, though somewhat complex, give some support to previous evidence that separate memory systems underlie the person impression and the verbal recall (Anderson \& Hubert, 1963).

Wyer (1970) obtained judgments of "desirability" of persons described by a trait adjective and an occupation noun. These data seem generally consistent with the present formulation, though some caution is needed in interpreting a dependent variable that apparently confounds likableness and social value. Wyer's own theoretical formulation employed two doubtful assumptions: that the integration process is additive and that the scale value of a trait is constant across nouns. The assumption of additivity is contrary to the present and previous data on the averaging hypothesis, though a direct test with adjective-noun combinations, similar to that used by Lampel and Anderson (1968), might still be desirable. The assumption of constant scale values seems quite doubtful for judgments of social value. If the weight parameter varies across nouns, as Wyer allows, it seems reasonable to expect that the value parameter will do the same.

\section{ADJECTIVE-NOUN INTERACTIONS}

So far, it has been assumed that words are integrated without interacting. By that is meant that their weights and scale values and, more generally, their meanings are the same in one combination as in another. This assumption requires scrutiny, since it might be expected that interactive changes of meaning would be common. Little definite evidence for change of meaning has been found, however, and various effects that might be so interpreted seem to have simpler interpretations when looked at more closely. Several different aspects of this issue need careful delineation.

Adjective-adjective combinations, judged on the dimension of likableness, have been studied extensively. Nearly all this work favors the hypothesis of no change of meaning (Anderson, 1971b, 1974); adjectives appear to retain the same scale values from one combination to another, which they would not do if their meaning changed. Mild changes in weight have been observed when marked inconsistency or redundancy was present. Such weight changes, however, though they certainly reflect interaction, are presumably not changes in meaning.

Adjective-noun combinations require more detailed consideration. For likableness judgments, the present data support the assumption of no stimulus interaction. Thus, the lack of significant adjective-noun interaction implies that humorous has the same scale value across all four occupations.

Humorous does seem to denote different behaviors for plumber than for lawyer. However, that may be merely a semantic illusion. Once combined or integrated, the adjective and noun are no longer separable; the seeming difference in adjective denotation may be just a direct effect of the noun (Anderson, 1971b).

Proficiency judgments for adjective-noun combinations present a somewhat different picture. The weight of any adjective will change from noun to noun, as has been illustrated above, and presumably the same holds for its scale value. This is certainly interaction of a kind, but it is important to recognize that it occurs in the valuation operation rather than in an integration operation. The noun merely defines the dimension of judgment, and the adjective represents a value on this dimension.

Furthermore, such changes in parameter values do not necessarily reflect changes in the denotation of the adjective. The weight and value of persuasive are different for judgments of proficiency at law than proficiency at plumbing. Yet persuasive may have the same meaning in both cases even though its implications are different.

Parenthetically, a similar remark applies to "implicit personality theory," which refers to the judgmental interrelations among personality traits: Each given trait constitutes a dimension of judgment, and any other trait will have a weight and scale value specific to that dimension of judgment. Likableness is only one of many such dimensions. Thus, interaction of a kind does occur here also, but again it is in the valuation operation that outputs the weight and value parameters (Anderson, 1973, Section IV.4).

Adjective-noun combinations can also involve inconsistency and redundancy. Thus, honest thief represents an inconsistency and so presents the hearer with a problem to solve. In ordinary discourse, this problem might be largely resolved by reference to the communicational context. Here, however, only the bare phrase will be considered.

One solution of honest thief is to discount (Anderson \& Jacobson, 1965). The hearer may partially deny the truth of one or both of the two pieces of information. Discounting reactions are likely when the situation allows for unreliability in the information. In terms of integration theory, discounting would correspond to changes in the weight parameter. But such uncertainty about the truth of the two pieces of information does not, presumably, imply that the meanings of the terms themselves are affected.

If both adjective and noun are to be taken as true, then some solution other than discounting seems needed. Since the problem arises from both terms being 
taken as general personality traits, it could, in the absence of contextual cues, be resolved by rationalizing. Thus, the hearer could invent information to ameliorate the inconsistency by claiming that the person was not basically a thief but had stolen out of great need or had stolen only an apple. On the other hand, the adjective could also be reinterpreted by claiming that the person was not basically honest, only in his dealings with other criminals.

These rationalizations would seem to reflect changes in meaning or value. By itself, thief presumably refers to some modal value in the distribution of thieves. The cited rationalizations can be represented as shifts away from the modal value toward less extreme values. Analogously, a redundancy like dishonest thief could be represented as a shift away from the modal value toward more extreme values.

Nevertheless, these interpretations may also correspond to simple noninteractive models. Indeed, both cited examples could be considered as simple averages. Alternatively, and more realistically, the adjective may correspond to a location on a dimension defined by the noun. Thus, a model might hold similar to that for adverb-adjective combinations (Anderson, 1974), augmented by a noun-specific zero point.

On these questions, there seems to be little information. The present survey is not intended to be complete, but it does indicate that adjective-noun interactions may be far less prevalent than expected. Indeed, if the valuation operation is excepted, it is not clear that there is at present any real evidence for such interactions. The above rationalizations of honest thief have an easy plausibility. However, similar armchair analyses of adjective-adjective combinations have failed to stand up to experimental analysis. This paper iepresents a start on a similar experimental analysis for adjective-noun combinations.

\section{REFERENCES}

Anderson, N. H. Integration theory and attitude change. Psychological Review, 1971a, 78, 171-206.

Anderson, N. H. Two more tests against change of meaning in adjective combinations. Journal of Verbal Learning \& Verbal Behavior, 1971b, 10, 75-85.

Anderson, N. H. Looking for configurality in clinical judgment. Psychological Bulletin, 1972, 78, 93-102.

Anderson, N. H. Cognitive algebra: Integration theory applied to social attribution. In L. Berkowitz (Ed.), Advances in experimental social psychology. Vol. 7. New York: Academic Press, 1973.

Anderson, N. H. Information integration theory: A brief survey. In D. H. Krantz, R. C. Atkinson, R. D. Luce, and P. Suppes (Eds.), Contemporary developments in mathematical psychology. Vol. 2. San Francisco: Freeman, 1974.

Anderson, N. H., \& Hubert, S. Effects of concomitant verbal recall on order effects in personality impression formation. Journal of Verbal Learning \& Verbal Behavior, 1963, 2, 379-391.

Anderson, N. H., \& Jacobson, A. Effect of stimulus inconsistency and discounting instructions in personality impression formation. Journal of Personality \& Social Psychology, 1965, 2, 531-539.

Birnbaum, M. H. The devil rides again: Correlation as an index of fit. Psychological Bulletin, 1973, 79, 239-242.

Chandler, J. P. Subroutine STEPIT-finds local minima of a smooth function of several parameters. Behavioral Science, $1969,14,81-82$

Gollob, H. F. Impression formation and word combination in sentences. Journal of Personality \& Social Psychology, 1968 , $10,341-353$.

Gollob, H. F., \& Lugg, A. M. Effect of instruction and stimulus presentation on the occurrence of averaging responses in impression formation. Journal of Experimental Psychology, $1973,98,217-219$.

Heise, D. R. Affectual dynamics in simple sentences. Journal of Personality \& Social Psychology, 1969, 11, 204-213.

Jaspars, J., Rommetveit, R., Cook, M., Havelka, N., Henry, P., Herkner, W., Pecheux, M., \& Peeters, G. Order effects in impression formation: A psycholinguistic approach. In E. A. Carswell and R. Rommetveit (Eds.), Social contexts of messages. New York: Academic Press, 1971.

Lampel, A. K., \& Anderson, N. H. Combining visual and verbal information in an impression-formation task. Journal of Personality \& Social Psychology, 1968, 9, 1-6.

Leon, M., Oden, G. C., \& Anderson, N. H. Functional measurement of social values. Journal of Personality \& Social Psychology, 1973, in press.

Oden, G. C., \& Anderson, N. H. Differential weighting in integration theory. Journal of Experimental Psychology, $1971,89,152-161$.

Osgood, C. E., Suci, G. J., \& Tannenbaum, P. H. The measurement of meaning. Urbana: University of Illinois Press, 1957.

Rokeach, M., \& Rothman, G. The principle of belief congruence and the congruity principle as models of cognitive interaction. Psychological Review, 1965, 72, 128-142.

Rosenberg, S. Mathematical models of social behavior. In G Lindzey and E. Aronson (Eds.), The handbook of social psychology. Vol. I. (2nd ed.) Reading, Mass: Addison-Wesley, 1968.

Shanteau, J. C., \& Anderson, N. H. Test of a conflict model for preference judgment. Journal of Mathematical Psychology, $1969,6,312-325$.

Warr, P. B., \& Smith, J. S. Combining information about people: Comparisons between six models. Journal of Personality \& Social Psychology, 1970, 16, 55-65.

Wyer, R. S. The prediction of evaluations of social role occupants as a function of the favorableness, relevance and probability associated with attributes of these occupants. Sociometry, 1970, 33, 79-96.

(Received for publication May 23, 1973; accepted May 29, 1973.) 